\title{
On the different contributions of coherent structures to the spectra of a turbulent round jet and a turbulent boundary layer
}

\author{
By T. B. NICKELS AND IVAN MARUSIC \\ ${ }^{1}$ Department of Engineering, University of Cambridge, \\ Trumpington Street, Cambridge CB2 1PZ, UK \\ ${ }^{2}$ Department of Aerospace Engineering and Mechanics, University of Minnesota, \\ Minneapolis, MN 55455, USA
}

(Received 13 November 2000 and in revised form 22 June 2001)

This paper examines and compares spectral measurements from a turbulent round jet and a turbulent boundary layer. The conjecture that is examined is that both flows consist of coherent structures immersed in a background of isotropic turbulence. In the case of the jet, a single size of coherent structure is considered, whereas in the boundary layer there are a range of sizes of geometrically similar structures. The conjecture is examined by comparing experimental measurements of spectra for the two flows with the spectra calculated using models based on simple vortex structures. The universality of the small scales is considered by comparing high-wavenumber experimental spectra. It is shown that these simple structural models give a good account of the turbulent flows.

\section{Introduction}

Measurements of spectra have been used as a tool for analysing fluid flows for many years now. They are particularly useful in periodic flows such as vortex shedding where peaks in the spectra can be identified with specific features of the flow. In fully turbulent shear flows the interpretation of spectra is more difficult. There is no question that the spectra contain important structural information but extracting this information is not straightforward. One reason is that a given wavenumber component does not necessarily reflect a particular structure (or 'eddy') in the flow. In fact a single structure can contribute energy across a wide range of wavenumber space. Also a given spectral signature does not uniquely reflect a given underlying structure in a flow.

In this paper a different approach to the interpretation of spectra is developed. In an effort to understand the structure of the flows we compare the experimental spectra with the spectra produced by random arrays of simple vortex structures. In this manner we are able to overcome some of the difficulties in the interpretation of spectra by comparing that of a known structure with that observed in the experiment. The aim is to examine to what extent the experimental results are consistent with an array of simple coherent structures. In particular the Reynolds shear-stress spectra (or cospectra) are examined. This component is useful since it does not contain contributions from locally isotropic motions that may be present at high wavenumbers.

One of the particular advantages of this work is that the measurements and the 
modelling have been conducted by the authors in very close collaboration. This means that the data collection and analysis is virtually identical for the two flows. It also means that the modelling is, as far as possible, very similar. As a result, it is possible to make meaningful comparisons between the two different flows. This is not always easy when dealing with published data produced by different researchers in different flows since there is always some uncertainty as to methods of analysis, measurement techniques and matching of appropriate parameters.

The results highlight essential structural differences and similarities between the two flows. They also give some general guidance as to the interpretation of the experimental spectra. Some preliminary aspects of the work presented here were given in Marusic, Nickels \& Perry (1994).

The conclusions of this paper regarding the structure of these two flows are in many ways consistent with more traditional arguments based on dimensional analysis and discussions of active and inactive motions in boundary layers and the different effects of the wall on the different velocity components in the flow. We aim to flesh out these arguments by conducting detailed modelling of the flows. It is one thing, for example, to say there are a range of scales of attached eddies in a boundary layer that explain the measurements, it is another to actually show that a 'real' array of eddies can reproduce the trends in the experimental data. Modelling in this way also identifies important necessary features in the structure that are not specified by the more general approach.

\section{Experimental apparatus and techniques}

The apparatus used for the jet flow and the boundary layer measurements are described fully in the respective theses of Nickels (1993) and Marusic (1991). Some details are also given in Nickels \& Perry (1996) and Marusic \& Perry (1995). Only the essential details will be given here.

In the case of the jet the wind tunnel has a cross-section of $1220 \times 890 \mathrm{~mm}$ with a maximum velocity of $3.5 \mathrm{~m} \mathrm{~s}^{-1}$ and a turbulence intensity of less than $0.6 \%$. The jet issues from a $25.4 \mathrm{~mm}$ diameter circular nozzle aligned with the wind-tunnel centreline. The nozzle has a contraction ratio of $9: 1$ which gives a turbulence intensity of less than $0.5 \%$ at the outlet. The exterior of the nozzle has perforations which allow boundary layer suction to avoid separation of the flow on the outer surface and allow the control of initial conditions. Flow in the jet is produced by a centrifugal blower through honeycomb and a series of screens. The jet flow could be varied from 2 to $35 \mathrm{~m} \mathrm{~s}^{-1}$ which allowed velocity ratios from 20 to 3 . The initial condition for the jet is close to a top-hat profile for all cases due to the external suction. Exact details may be found in Nickels \& Perry (1996).

In the case of the boundary layer the wind tunnel is of an open-return blower type. It consists of a contraction with area ratio $8.9: 1$ leading to the inlet of a $4.3 \mathrm{~m}$ long working section with a cross-section measuring $940 \times 388 \mathrm{~mm}$. The free-stream velocity at the inlet of the working section can be varied between 2 and $35 \mathrm{~m} \mathrm{~s}^{-1}$, while the free-stream turbulence intensity is less than $0.3 \%$. The working section consists of a smooth wall made from a polished acrylic laminate and a series of louvres (or bleeding vanes) which make up the roof. The working section is operated above atmospheric pressure and the streamwise pressure gradient is adjusted by controlling the location and amount of air leaving the working section through the louvres. For the data to be presented in this paper the streamwise pressure gradient was set to be nominally zero. Spanwise $x$-wire surveys of the spanwise velocity component 
confirmed the flow to be nominally two-dimensional in the mean. The boundary layer is tripped at the beginning of the working section $(x=0)$ with a trip wire sized such that $U_{1} D / v=800$ where $U_{1}$ is the free-stream velocity, $D$ is the wire diameter and $v$ is the kinematic viscosity. This is based on the recommendation of Erm \& Joubert (1991) for correctly tripped turbulent boundary layers.

All turbulence measurements in both flows were made with $90^{\circ} \times$-wires constructed with $5 \mu \mathrm{m}$ diameter platinum Wollaston wire, etched approximately to $1.0 \mathrm{~mm}$. Constant-temperature hot-wire anemometers were used and operated at a resistance ratio of 2.0.

Power spectral densities were calculated from dynamically matched but uncalibrated $x$-wire signals by using an FFT-algorithm. The analogue dynamic matching leads to two voltage outputs each of which is sensitive to only one velocity component. Tests here and in previous work suggested that there is no significant difference in the spectra taken with calibrated and uncalibrated probes. The signals were sampled at three different sampling rates to improve the frequency bandwidth of the spectrum at low frequencies and were low-pass filtered at less than half the digital sampling rate to avoid aliasing of the measured spectrum (using fourth-order Butterworth filters). A number of 'records' of data were taken and the spectra were ensemble-averaged. Typically 30 to 100 records were taken, and the spectra averaged, to form the final spectra files. The three resulting spectral files (resulting from the three sampling rates) were then joined to form a single spectral file by dropping points at the high end above $70 \%$ of the filter cut-off frequency (that would be attenuated) and points at the low end which were not well-resolved. The resulting file was then minimally smoothed with a simple three-point smoothing. To transform the spectral argument from frequency, $f$, to streamwise wavenumber, $k_{1}$, Taylor's hypothesis of frozen turbulence was used, i.e. $k_{1}=2 \pi f / U_{c}$, where $U_{c}$ is some local convection velocity assumed (in this case) to be equal to the local mean velocity of the flow at the point of interest. The spectra were normalized so that

$$
\int_{0}^{\infty} \phi_{i j}\left(k_{1}\right) \mathrm{d} k_{1}=\overline{u_{i} u_{j}}
$$

where $\overline{u_{i} u_{j}}$ is the appropriate component of the Reynolds stress tensor and $\phi_{i j}$ is the corresponding one-dimensional power spectral density. It should be noted that, with this convention, repeated subscripts do not denote a summation. In addition, it is important to note that by definition $\phi_{i j}\left(k_{1} l\right)$ represents the power spectral density per unit non-dimensional wavenumber $k_{1} l$; which implies

$$
\phi_{i j}\left(k_{1} l\right)=\frac{\phi_{i j}\left(k_{1}\right)}{l}
$$

where $l$ is some length scale of interest. In this paper the co-ordinate convention is such that the streamwise $(x)$ fluctuating velocity is $u_{1}$ and the cross-stream (wall-normal, $z$, for boundary layers and radial, $r$, for jets) component is $u_{3}$.

In order to determine the Kolmogorov length and velocity scales (used in the latter part of the paper) an estimate of the dissipation rate is required. Here the isotropic assumption is used with Taylor's hypothesis, giving

$$
\epsilon=15 v \int_{0}^{\infty} k_{1}^{2} \phi_{11}\left(k_{1}\right) \mathrm{d} k_{1},
$$

for want of a better method. The Kolmogorov length and velocity scales are given 


$\begin{array}{cccc}K_{\tau} & 1229 & 3290 & 4412 \\ R_{\theta} & 2740 & 8190 & 11100\end{array}$

TABLE 1. Values of $R_{\theta}$ corresponding to values of $K_{\tau}$ presented.

(respectively) by

$$
\eta=\left(\frac{v^{3}}{\epsilon}\right)^{1 / 4}
$$

and

$$
v=(v \epsilon)^{1 / 4} .
$$

Another reference parameter that is useful when considering the high-wavenumber spectra is the Taylor microscale Reynolds number. Whilst the physical interpretation of this parameter is problematic in turbulent shear flows, it is commonly used in discussions of isotropic turbulence, and hence provides some point of reference for comparison. In this paper it is calculated using the isotropic assumption which gives the Taylor microscale as

$$
\lambda_{T}^{2}=15 v \frac{\overline{u_{1}^{2}}}{\epsilon}
$$

and the Reynolds number

$$
R_{\lambda}=\overline{u_{1}^{2}} \sqrt{\frac{15}{\epsilon v}}
$$

\section{Results and discussion}

\subsection{Reynolds shear-stress spectra}

Before discussing the differences in structure of the two flows it is important to have a basis for comparison. It is necessary to define an appropriate Reynolds number and also to define an appropriate shear layer thickness so that different cross-stream positions through the layer may be compared. In boundary layer work a useful Reynolds number is the Kármán number $K_{\tau}=\delta_{c} U_{\tau} / v$ where $\delta_{c}$ is the boundary layer thickness and $U_{\tau}$ is wall shear velocity. The boundary layer thickness is determined by the method given in Perry \& Li (1990) and relies on least-squares-error fitting a functional form to the mean velocity profile. For jets an equivalent Reynolds number based on local conditions can be defined as $R_{\Delta}=\Delta U_{o} / v$ where $U_{o}$ is the local velocity excess (i.e. velocity on centreline minus external velocity) and $\Delta$ is the standard deviation of the mean velocity profile. These definitions are based on measurements of the mean flow. In order to compare different cross-stream positions, a relationship between $\delta_{c}$ and $\Delta$ needs to be established. If we consider the edge of the turbulent zone to be the point where the Reynolds shear stress has fallen to zero (or some small value) and call this $\delta_{S S}$ then $\delta_{c} / \delta_{S S} \approx 1$ and $\delta_{S S} / \Delta \approx 3$. Wherever possible, positions with similar $z / \delta_{S S}$ and $r / \delta_{S S}$ have been shown (although legends in the figures give $r / \Delta$ and $z / \delta_{c}$ values in all cases). Unfortunately, an equivalent factor relating $U_{o}$ and $U_{\tau}$ is unknown and hence it is not possible to directly relate the Reynolds numbers between the two flows. This does not seriously affect any of the conclusions drawn in the paper. When comparing the high-wavenumber spectra we use the Taylor microscale Reynolds number. Since not all researchers make use of 

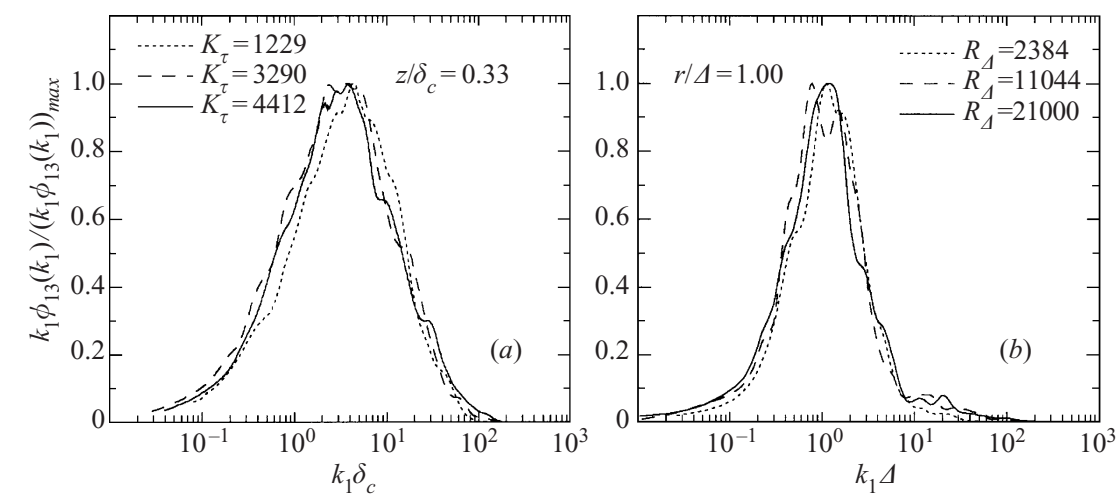

FIGURE 1. Comparison of effect of Reynolds number on the form of premultiplied Reynolds shear-stress spectra at a fixed cross-stream position: $(a)$ boundary layer, $(b)$ jet.

the Kármán number we have included table 1 that shows the values of momentum thickness Reynolds number $\left(R_{\theta}\right)$ that correspond to the Kármán numbers presented.

In order to examine the effect of changes of Reynolds number on the wavenumber extent of the Reynolds shear-stress spectra $\left(\phi_{13}\right)$, figure 1 shows the premultiplied Reynolds shear-stress spectra for several Reynolds number cases for each flow at a fixed cross-stream position. Since we are only interested in the wavenumber extent, or bandwidth, of the spectra they have been divided by their maximum values. Hence we can see the change of shape without regard to the actual magnitudes.

It can be seen that the bandwidth does not seem to change significantly for either the jet or the boundary layer flow. This suggests that the large-scale structures contributing to the Reynolds shear stress may be considered to be essentially inviscid.

Figure 2(a) shows the change in the bandwidth of the Reynolds shear-stress spectra at a fixed Reynolds number for varying cross-stream positions. It may be seen that as the wall is approached the bandwidth increases for the boundary layer flow but remains substantially unchanged for the jet flow. This suggests that, as the wall is approached, the range of scales which contribute to the Reynolds shear stress increases for the boundary layer. In particular the contribution from higher wavenumbers increases. Conversely as the measurement position moves further from the wall the bandwidth for the boundary layer decreases and it is interesting to compare the spectra in the outer part with the jet. This is shown in figure 3. It may be noted that the bandwidth of the spectrum is very similar to that of the jet. In fact the form of the two is so similar it is difficult to differentiate between them. This suggests that the structure of the outer part of the turbulent boundary layer (for the Reynolds-stress-producing motions) is quite similar to that of the turbulent jet. It should be noted of course that signs of the vorticity in the two cases are different.

\subsection{The physical models}

It is important to emphasize the objective of the models before proceeding: it is to examine to what extent a random array of simple inviscid vortex structures can reproduce the trends observed in the measured spectra. Comparison of these simple models with experiments helps us to identify the essential features of the underlying structure necessary to reproduce the important experimental observations. Two related vortex models are used to examine and explain the trends in the measurements. Mathematical descriptions of the models for the boundary layer and jet are given in detail by Perry \& Marusic (1995) and Nickels \& Perry (1996) respectively. They 


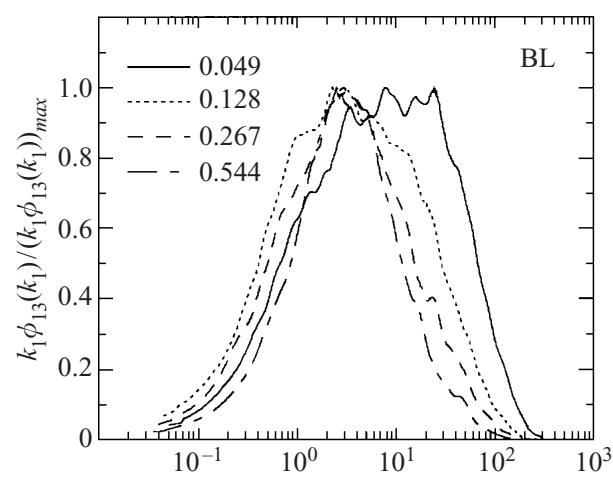

(a)

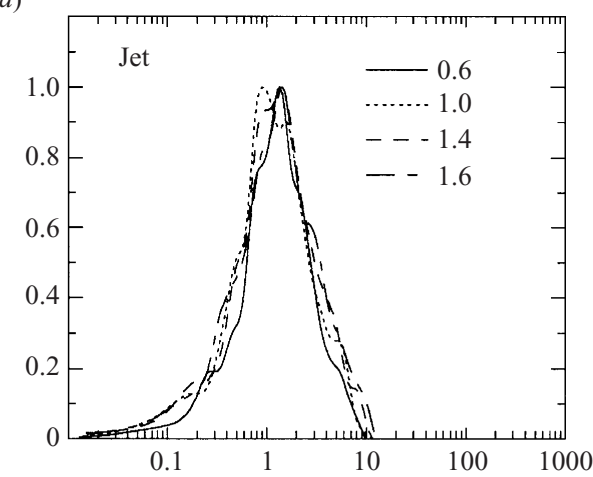

(b)
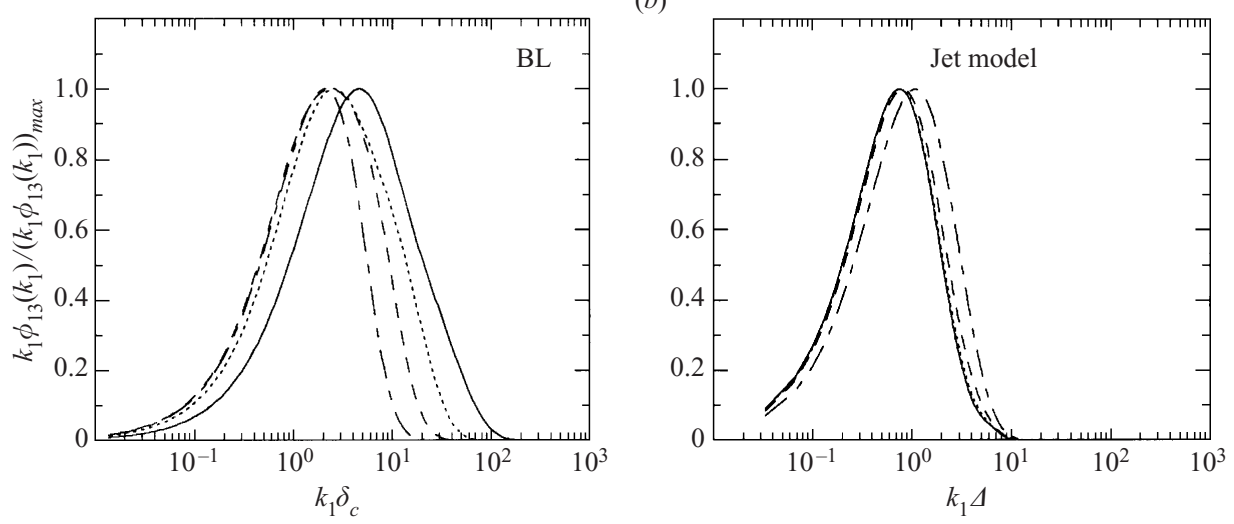

FIGURE 2. Comparison of variation of the bandwidth of the premultiplied Reynolds shear-stress spectra at a fixed Reynolds number with cross-stream position $\left(z / \delta_{c}\right.$ for boundary layer $(\mathrm{BL})$ and $r / \Delta$ for jet). Note the increase in bandwidth for the boundary layer as the wall is approached. (a) Experimental results, $K_{\tau}=\delta_{c} U_{\tau} / v=4412$ for BL; $R_{\Delta}=2384$ for jet, (b) results from coherent structure models developed by the authors for same positions as in $(a)$.

have the same underlying basis but differ in some important details. The basis of the models is that a given turbulent flow can be kinematically modelled by a series of geometrically similar vortex structures or 'typical eddies' arranged randomly in space. In both models the typical structures are constructed from a number of joined, straight vortex rods: this speeds up and simplifies calculations. These rods have a Gaussian distribution of vorticity within their cores to avoid singularities in the velocity field. The velocity field produced by one such structure can be calculated by applying the Biot-Savart integral over a region of space. The spectral signatures of the eddies can then be calculated and ensemble-averaged in order to account for a random array of geometrically similar but uncorrelated eddies. Doing this is equivalent to using Campbell's theorem (see Papoulis 1965) for uniform-density shot-noise distributions. In the boundary layer the eddies are considered to be randomly arranged on a plane and in the (axisymmetric) jet they are randomly arranged around the surface of a cylinder.

Using convolution integrals it is then possible to account for a random spatial 'jitter' of the structures (as in the jet) and a distribution of geometrically similar eddies of different sizes and population densities (as in the boundary layer).

It is in this latter part that the two approaches differ. In the jet model there is 


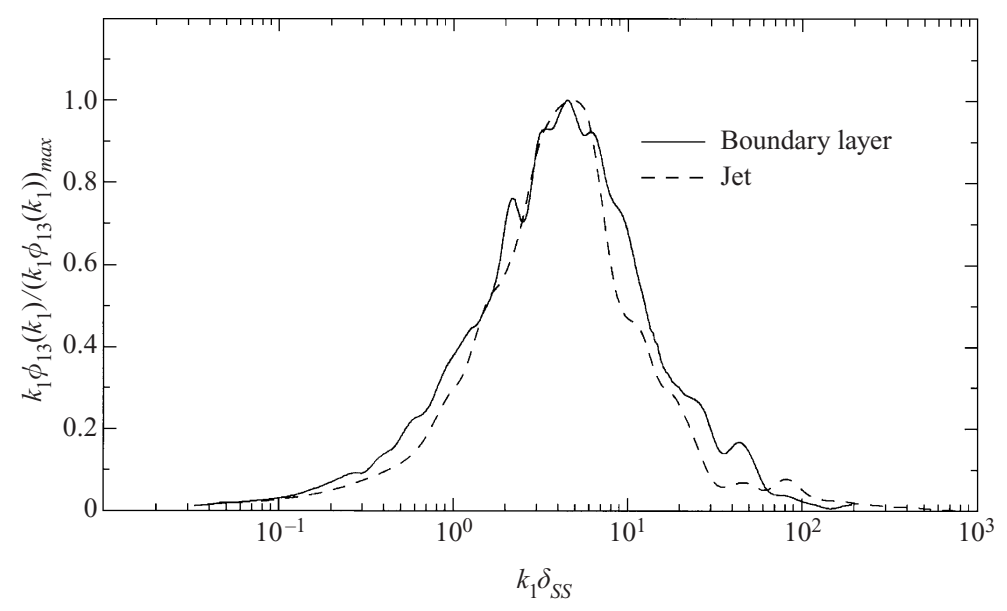

FIGURE 3. Spectrum from the outer part of the turbulent boundary layer compared to the spectrum from a typical level of the jet. Boundary layer profile corresponds to $z / \delta_{c}=0.93$ and jet to $r / \Delta=1.5$.

a random array of one typical eddy of one size to which spatial jitter is applied. The spatial jitter is essentially a random displacement of the structure about a mean position relative to the jet centreline. The random displacement is confined to a cross-stream plane (i.e. $x=$ constant): there is no jitter in the streamwise direction. If we consider a Cartesian coordinate system then the eddy has a mean position in the $(y, z)$-cross-stream plane and then has a random displacement in both directions based on a Gaussian distribution. The variance of this displacement is the same in both the $y$ - and $z$-directions and hence is specified by one parameter. It is dealt with by a convolution integral. The effect is to average nearby spectral signatures, creating smoother spectral curves. This jitter can be physically, and quantitatively, related to the intermittency of the outer region of the jet. Conceptually, the mean position of the eddy is related to the mean position of the turbulent/non-turbulent interface and the amount of jitter is related to the variance of the interface about this mean position. In this conceptual framework it is the movements of the large 'typical eddies' (within the background fine-scale turbulence) that lead to the distortions of the interface. The values for mean position of the eddies and the amount of jitter are made consistent with measurements of the turbulent/non-turbulent interface in jets (see Nickels \& Perry 1996).

In the case of the boundary layer a range of sizes of geometrically similar eddies is considered, with different sizes of eddies having different population densities which are distributed approximately as an inverse-power-law PDF with the eddy size, i.e. the number of small eddies is much larger than the number of large eddies. The structure of these models is partly inspired by various observations of the structure of the two flows and, in the case of the boundary layer, the ideas of Townsend (1976) as developed by Perry \& Chong (1982). In the Perry \& Marusic (1995) attached eddy model two types of representative structures are used. The first is a wall structure where the vortices are hairpin-shaped and extend to the wall, and the second is a wake structure where the vortices do not extend down to the wall but undulate in the cross-stream direction. The two components are assumed to be statistically uncorrelated and hence the contributions from each can be linearly added. In this way, the formulation provides a physical basis for the Coles (1956) law of the wall and law of the wake which has proven to be a reliable description of the mean velocity 


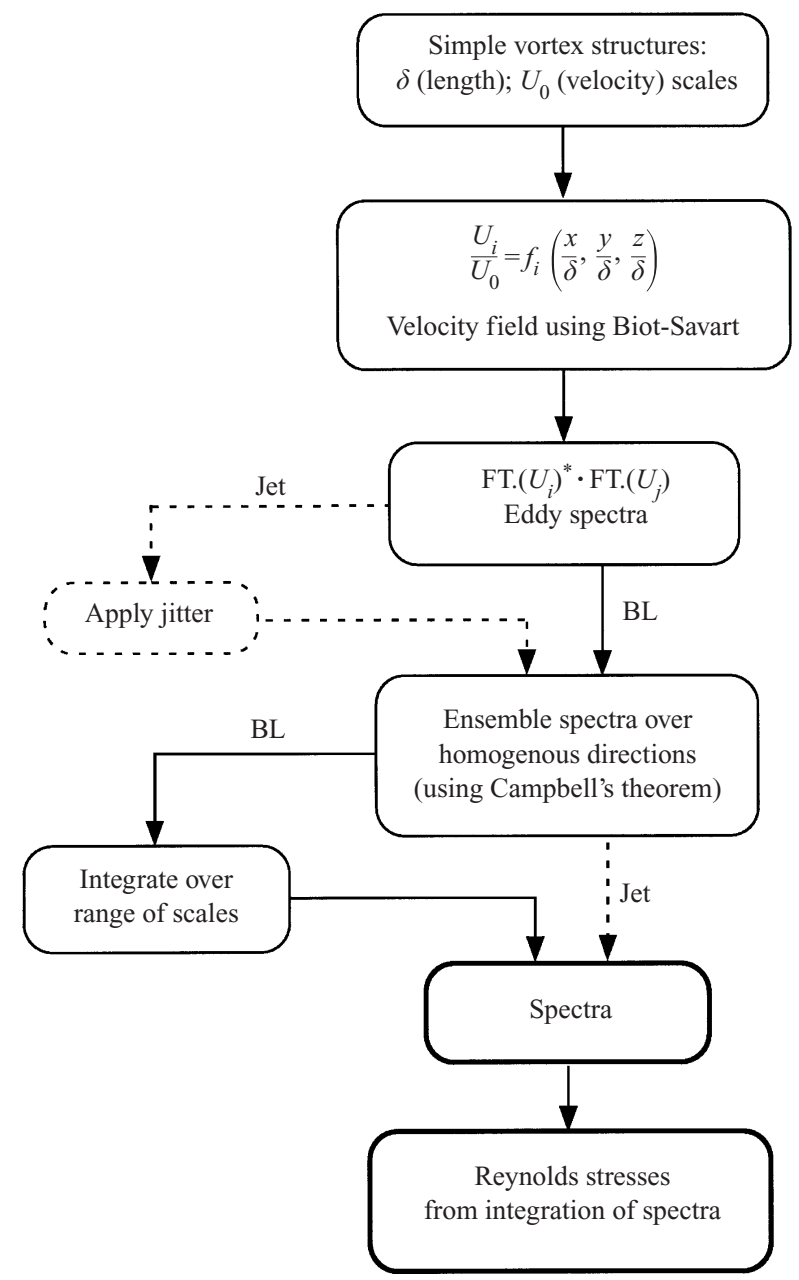

FiguRE 4. Procedure for calculating spectra from models.

profile for flows in a great range of pressure gradients. The wake structures are distributed with a PDF such that the overall result is similar to a single scale of wake eddy. This approach has been used since it is similar to that used for wall-attached eddies and so is a convenient technique for their inclusion. However, considering the similarity of the outer-flow co-spectra shown in figure 3, together with the success of the spatial jitter in the jet and its conceptual (and quantitative) link to intermittency, it would seem that using a jittered single-length-scale eddy may be a more appropriate way to deal with the wake structure. This avenue is being investigated by the authors. In any case, in zero-pressure-gradient flows such as the ones considered here, the wake component is small and the hairpin-type (wall) structure is dominant, except for the outermost region of the boundary layer.

The models produce results for the spectral components, the Reynolds shear stresses (via integration of the spectra) and the mean flow. Figure 4 is a block diagram outlining the overall calculation procedure.

These models then represent essentially different structures for the two flows. In the jet a single scale of typical eddy determines the spectra whereas in the boundary 
(a)
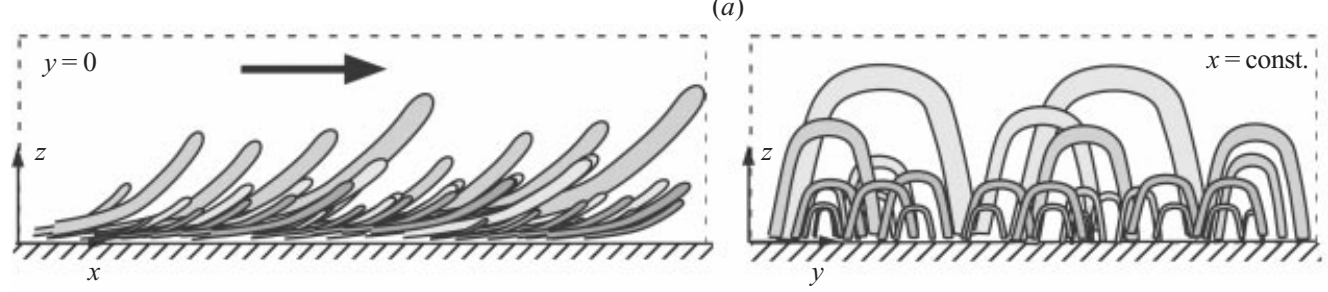

(b)
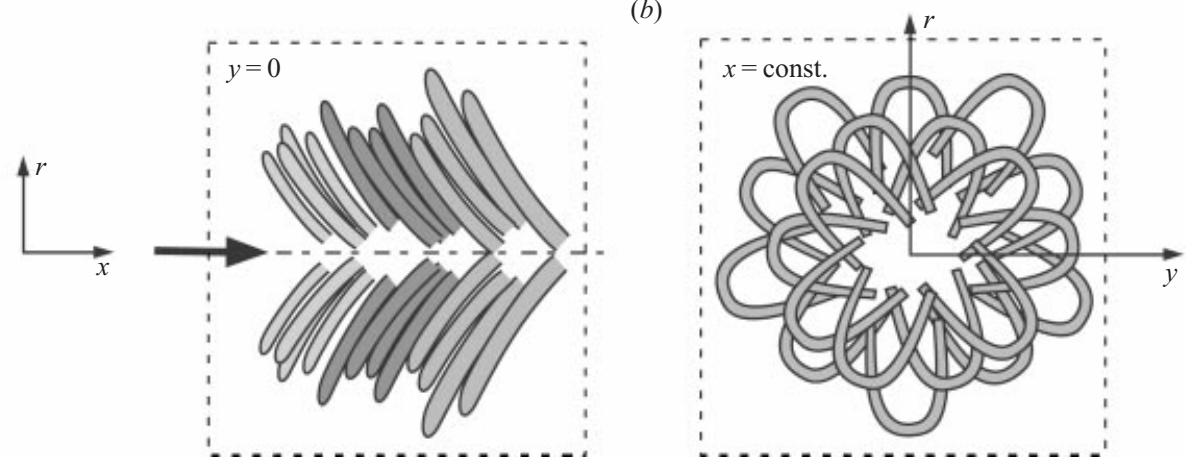

FIGURE 5. Proposed structure of $(a)$ the turbulent boundary layer and $(b)$ the jet. Here, for clarity, the eddies shown are only the ones associated with the sectional planes indicated and only hairpin wall eddies are shown for the boundary layer.

layer a range of scales of similar eddies is responsible for the measured spectra. Figure 5 shows cartoons of the structures of both flows. In the case of the boundary layer structure only hairpin-type wall structures are shown since these dominate the zero-pressure-gradient flow. The diagrams are, of course, simply sketches of the basic structure: in reality the flow is more complex due to the random positioning of the eddies and distortions of the basic structure. It is important to realise that the similar eddies shown are 'typical' (representative) eddies and hence do not necessarily correspond with any one individual eddy that occurs in the flow. They can be thought of as a statistically averaged eddy shape that captures the essential common features of most of the structures occurring in the real case. The basic shapes are inspired by various experimental observations of the flows.

\subsection{Free parameters in the models}

As with any model there are some free parameters available in these two models. Before detailing these parameters it should be stressed that, at this stage, the models are not meant to be predictive. The question we are addressing is 'Is it possible to reproduce experimental trends using an array of simple, geometrically similar, coherent vortex structures'. The answer to this question is not obvious, particularly as we require the models to produce the correct trends for the mean velocity, all components of the Reynolds stress and the spectra. In this paper we are particularly concerned with the spectra but the eddies used also must satisfy the other constraints. We also wish to show how the construction of these models leads to conclusions about the structural differences between the two flows.

The eddy shapes used are shown in figure 6 . In the case of the jet there are five vortex rods joined to form a symmetrical structure. In order to specify this structure we need 

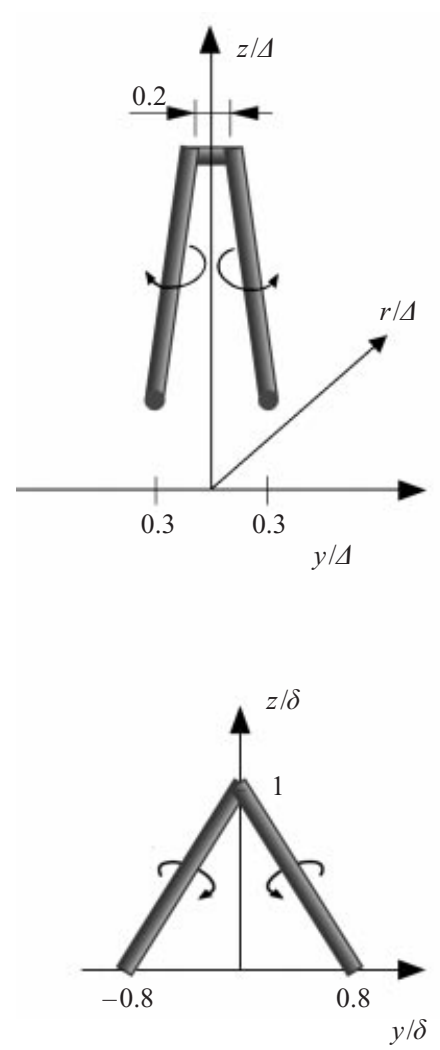

(a)

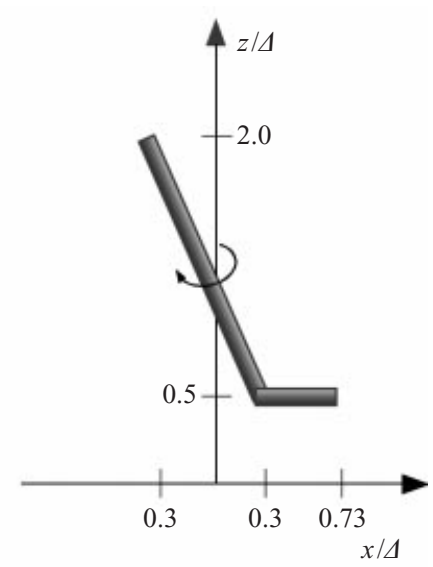

(b)

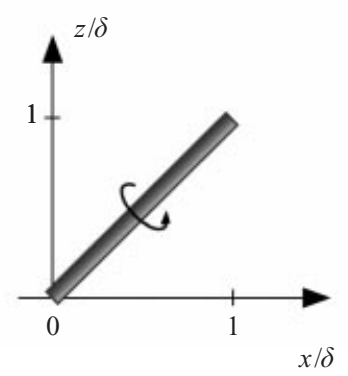

FIGURE 6. Details of 'typical eddies' used in the models. (a) Jet, (b) boundary layer.

three triplets in three-dimensional space to denote the positions at which the rods join. In the case of the boundary layer we require only two. Only the 'type-A' wall-structure vortices are shown. Type-B wake eddies are similarly very simple vortices made up of an ensemble of ' $\mathrm{V}$ '- and ' $\Lambda$ '-shaped eddies which do not extend below $z / \delta=0.5$. As mentioned earlier, type-B eddies have a significant role in adverse-pressure-gradient boundary layers but only a minor role in the zero-pressure-gradient boundary layer considered here.

The choice of the constants for the eddy shapes in figure 6 is constrained by the requirements that the mean flow, the spectra and the four significant components of the Reynolds stresses are comparable with the real flow. In addition the shapes are chosen so as to be consistent with observations of structures in both flows. It is important to note that the results presented are not strongly sensitive to the choice of eddy shape.

The real strength of this approach is that the eddies provide a direct and physical link between the mean flow, the Reynolds stresses and the spectra. Apart from eddy shape details, the only remaining parameters are the distribution of eddies in the case of the jet, and the population density and weighting applied for different scales of eddies for the boundary layer. In the case of the jet this corresponds to a 'jitter' parameter which involves a random displacement of the eddy from its mean position. The value of this parameter was chosen to be consistent with the displacement of the interface found from intermittency measurements and is hence constrained. In the case of the boundary layer the weighting functions are determined uniquely from 
the mean velocity flow field and the equations of motion. The procedure involves using the mean momentum and continuity equations to solve for the Reynolds shear stress which yields the weighing functions from a deconvolution calculation. No further parameters are available for adjustment.

If all of these parameters were simply arbitrary constants in a turbulence model then it would seem possible to fit any set of results. In these models the choices are quite seriously constrained by the physics of the vortex structures and the requirement of having a simple, plausible coherent structure. The constants are also not independent since changing the eddy shape affects all of the results in a nonlinear fashion.

\subsection{Vortex model results}

The results for the Reynolds shear-stress spectra from the models are shown in figure $2(b)$. It may be seen that there is good qualitative agreement with the experimental behaviour shown in figure $2(a)$. The candidate eddy shapes used are those shown in figure 6, that is, the same as were used in Perry \& Marusic (1995) and Nickels \& Perry (1996). Further refinement of the eddy shape would improve the quantitative agreement with experiment but this is not the objective of the present study. In any case, these results give further support to the basis for the structure of the models, namely: with a decrease in $z / \delta_{c}$ in the boundary layer, the bandwidth in $k_{1} \phi_{13}\left(k_{1}\right)$ increases whereas for the jet the bandwidth remains essentially constant as $r / \Delta$ is decreased. The reason for the increase in bandwidth for the boundary layer as the wall is approached may be understood by referring to the 'cartoon' of the flow. A probe at a given distance from the wall will only be significantly influenced by eddies the same size as or larger than this distance. As the wall is approached the probe experiences contributions from more eddies-in particular smaller eddies-leading to an increase in the energy at high wavenumbers. It should be noted that the smallest size of eddy is determined by the viscous length scale, $v / U_{\tau}$ (following Kline et al. 1967), and hence there will be a limit as the wall is approached beyond which there will be no further increase in bandwidth. In the jet there is only one basic scale of coherent structure across the whole width and hence the bandwidth does not change significantly with radial position (though the detailed shape of the spectral signature may change). One possible explanation for the difference in the range of scales in the two flows is that the vorticity in the jet is generated only at the nozzle, whereas in the boundary layer new vorticity can be continually generated at the wall as the flow develops.

\subsection{Streamwise spectra}

The structure of the flows is also reflected in the streamwise spectra. One reason for concentrating on the Reynolds shear-stress spectra is that this component has no contribution from fine-scale locally isotropic motions. Here we are most interested in the large-scale coherent motions. It is, nevertheless, instructive to examine the changes in the streamwise spectra. Figure 7 shows the streamwise spectra for both flows cases at different Reynolds numbers; also shown on the same plots are the Reynolds shear-stress spectra for these cases. The important feature to notice is that the streamwise spectra show an increase in energy at the high-wavenumber end with the increased Reynolds number. This does not occur in the Reynolds shear stress spectra. This observation is consistent with the idea of large-scale motions in the flow contributing to the Reynolds shear stress combined with finer-scale, locally isotropic motions that contribute to the other components. These motions are considered in 

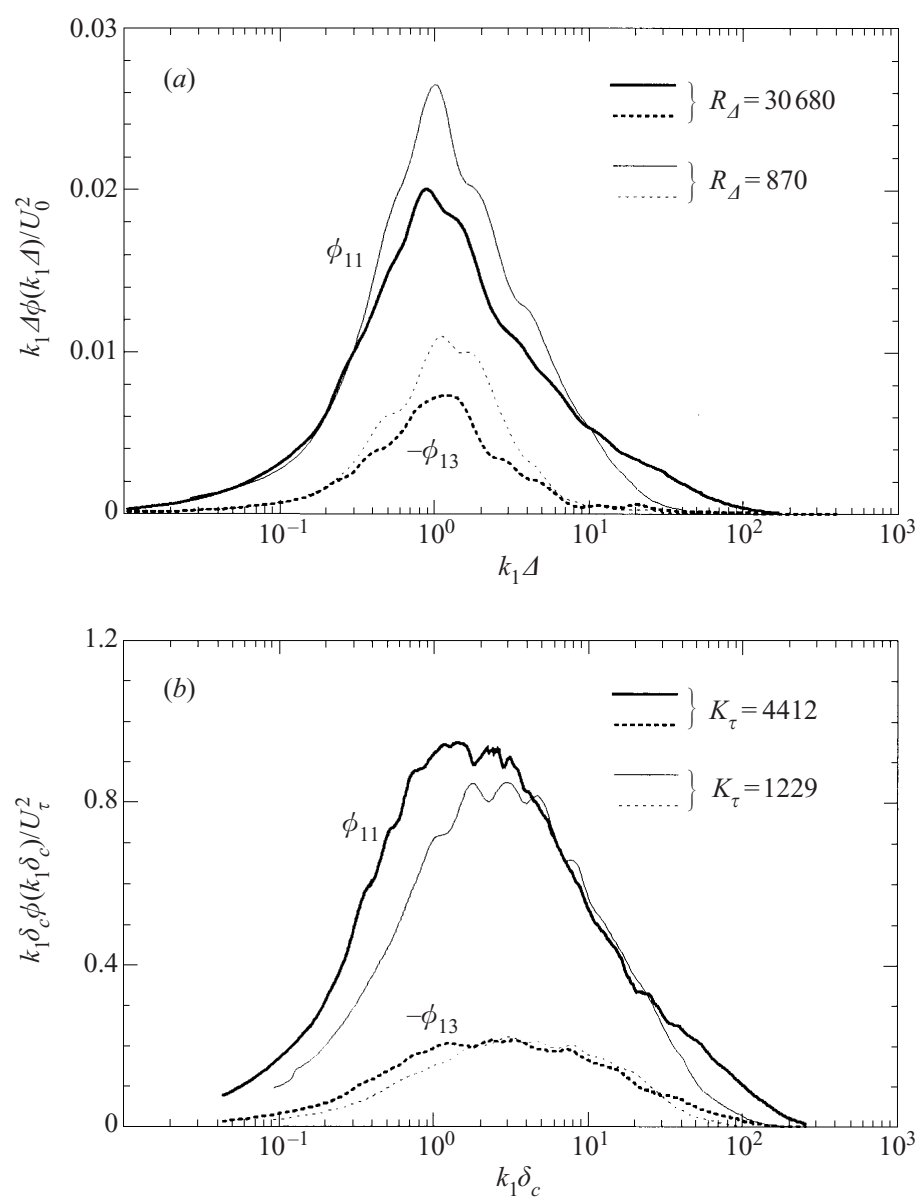

FIGURE 7. Comparison of the effect of the Reynolds number on the form of premultiplied streamwise and shear-stress spectra at a fixed cross-stream position. (a) Jet profiles at $r / \Delta=1.2$. ( $b$ ) Boundary layer profiles at $z / \delta_{c}=0.168$ which is within the logarithmic region for this flow.

the next section. It should be noted that these motions are not included in either of the models.

To summarize the major difference between the jet and boundary layer streamwise spectra, a schematic comparison is shown in figure 8. The main point is that for the jet, the spectrum consists of the component from a single scale of coherent structure plus the high-wavenumber contribution from the fine-scale locally isotropic motions. The boundary layer consists of a sum of contributions from different size coherent motions and the contribution from the fine-scale locally isotropic motions. The contribution from the locally isotropic motions is shown as the hatched region on the diagrams.

Also shown in figure 8 is the approximate range of wavelengths $\left(\Lambda=2 \pi / k_{1}\right)$ which are involved. The quantitative values given are based on the experimental data. As an example, figure 9 shows the streamwise spectra profiles across the boundary layer for $K_{\tau}=4412$. It is seen that peak streamwise turbulence intensity contributions come from large streamwise wavelengths corresponding to approximately $k_{1} \delta_{c}=1$ for positions within the logarithmic region of the flow (defined tentatively as $z_{+}>$ $\left.100 ; z / \delta_{c}<0.2\right)$. 

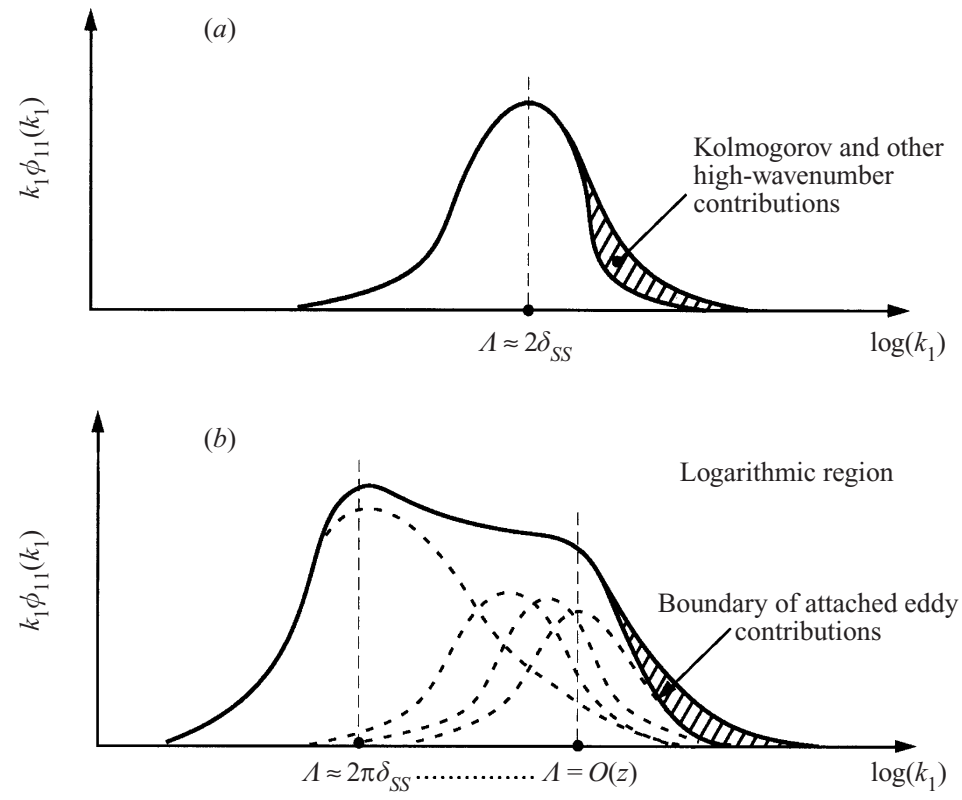

FIGURE 8. Sketch showing proposed composition of the streamwise spectra for (a) jet and $(b)$ boundary layer flow cases.

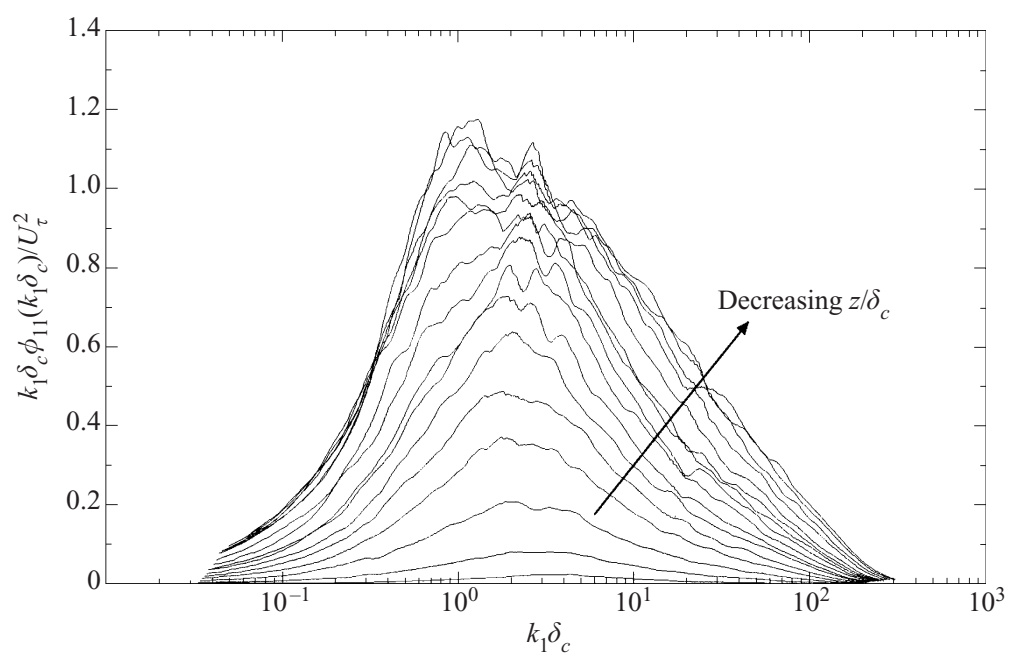

FIGURE 9. Premultiplied streamwise spectra across the turbulent boundary layer for $K_{\tau}=4412$. Positions correspond to: $z / \delta_{c}=0.049,0.069,0.095,0.128,0.168,0.214,0.267,0.326,0.392,0.465$, $0.544,0.630,0.723,0.822,0.928$.

The above observations of long streamwise energetic contributions relate directly to recent studies by Adrian, Meinhart \& Tomkins (2000), Zhou et al. (1999) and Kim \& Adrian (1999), who have proposed that boundary layer hairpin vortices most commonly appear in spatially correlated packets or trains of vortices, which can extend over many boundary layer thicknesses in length. No attempt has been made to incorporate these concepts in the attached eddy model presented here, as the outcome 
would not alter the qualitative trends being proposed. This aspect of the attached eddy modelling work will be considered in Marusic (2001).

Another notable issue concerning the streamwise spectra and co-spectra in boundary layers is whether or not a -1 power-law exists. Perry, Henbest \& Chong (1986) argued using the attached eddy hypothesis of Townsend (1976), that the streamwise spectra will have a -1-law at the main energy-containing wavenumbers for positions in the layer where the logarithmic law of the wall holds. This would show up as a plateau region in the premultiplied spectra. Using the same theoretical arguments as Perry et al. (1986) leads one to conclude that streamwise and spanwise spectra will show a -1 power law, but not the wall-normal spectra or co-spectra. Calculations using the attached eddy model have confirmed this. Results for co-spectra are shown in figure 2. Results for streamwise spectra were shown in Marusic \& Perry (1995). The calculations also indicate that very high Reynolds numbers (far beyond the present experiments) are needed to show a convincing -1-law for $\phi_{11}$. This is consistent with the experimental data in figure 9 where only weak evidence of a plateau region is observed for positions within the logarithmic layer.

\subsection{Local isotropy and Kolmogorov scaling}

In order to examine the universality of the fine scales in the two flows, spectra were measured and compared. If the high wavenumbers are universal then it would be expected that the spectra from all flows should collapse when non-dimensionalized with the Kolmogorov length and velocity scales. Figure 10 shows a range of typical streamwise spectra from the two different flows scaled with this Kolmogorov scaling. It may be seen that all profiles shown from both flows seem to collapse onto a single curve in the high-wavenumber region. There is however some peel-off from this universal form at the highest wavenumbers which is probably due to the limit of the spatial resolution of the hot-wire sensor. This explanation for the peel-off is supported by the fact that the spectra peel off at values of $k_{1} \eta$ that correspond to $k_{1} \ell=1$ where $\ell$ is the length of the hot-wire sensor. The limited length of the sensor acts as a spatial filter which attenuates all scales smaller than $\ell$ (i.e. $k_{1} \ell>1$ ).

Figure $10(b)$ shows the same streamwise spectra premultiplied by $\left(k_{1} \eta\right)^{5 / 3}$. On this plot a $-5 / 3$ power-law in the spectrum appears as a plateau. The diagram shows a short region which appears to be of $-5 / 3$ power-law form. It is also possible from this plot to estimate the Kolmogorov constant for the one-dimensional spectrum $\left(K_{o}\right.$ where $\left.\phi_{11}\left(k_{1} \eta\right) / v^{2}=K_{o}\left(k_{1} \eta\right)^{-5 / 3}\right)$. Here it appears to be approximately $0.5<K_{o}<0.6$ which is close to the value found in various other flows. Sreenivasan (1995) suggests a value of $K_{o}=0.5 \pm 0.05$, based on a compilation of existing data.

The arguments of Kolmogorov also suggest that the universal form for the spectra should correspond to a region of local isotropy. Since we have shown that the streamwise spectra appear to be universal it is worthwhile examining the existence of local isotropy in the flows. There is no definitive way to check for local isotropy experimentally but there are a number of options. Here we have chosen one of the simplest. We will examine the Reynolds shear-stress correlation coefficient defined as

$$
R_{13}\left(k_{1} \eta\right)=-\frac{\phi_{13}\left(k_{1} \eta\right)}{\sqrt{\phi_{11}\left(k_{1} \eta\right) \phi_{33}\left(k_{1} \eta\right)}} .
$$

Since there can be no shear-stress component in isotropic turbulence then $R_{13}$ should go to zero if the flow becomes locally isotropic. Figure 11 shows the premultiplied spectra and the corresponding plot of $R_{13}$ taken at a single value of $R_{\lambda}$ (Taylor microscale Reynolds number) for both flows. Two points are worth noting. First, the 

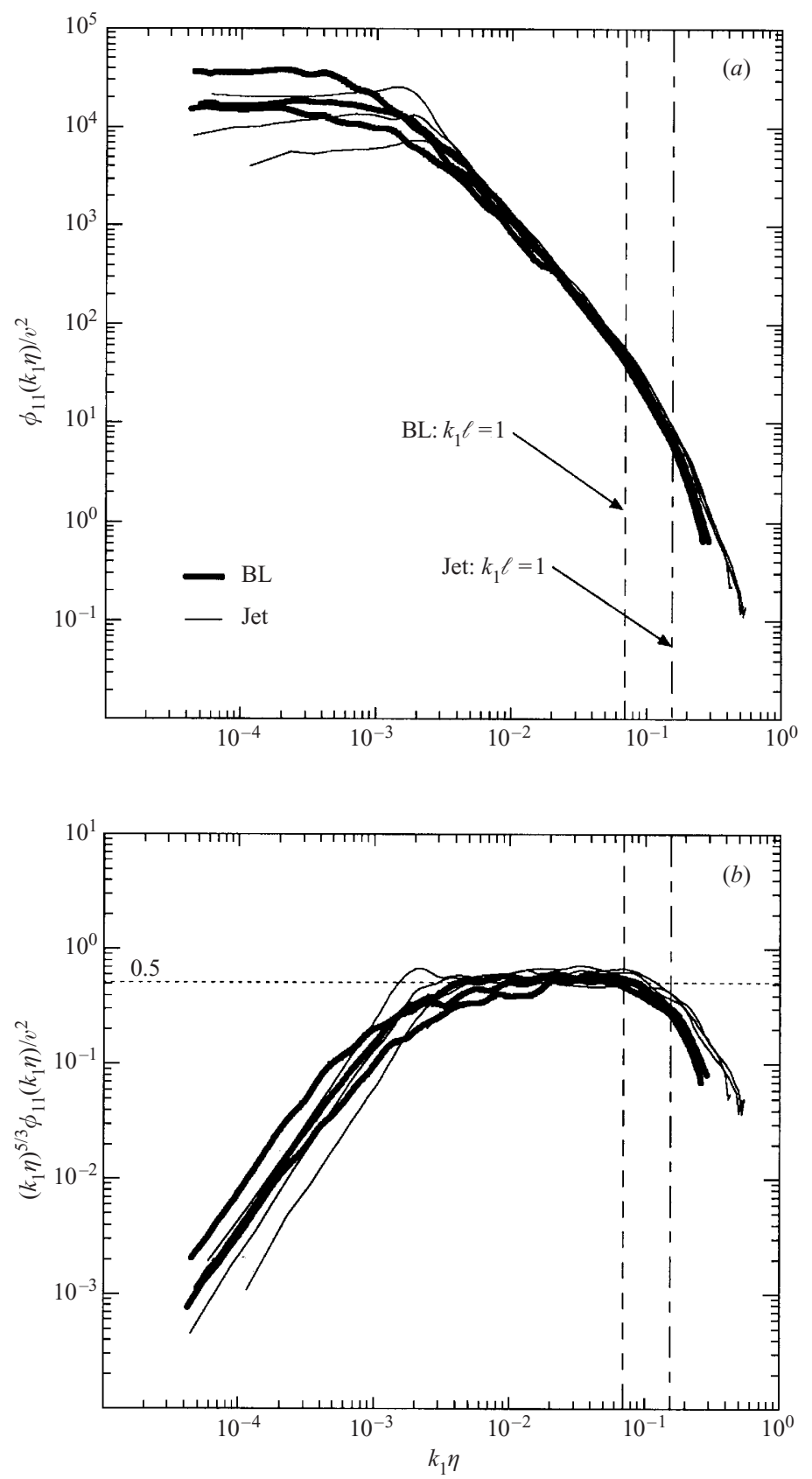

Figure 10. (a) Collapse of data for the jet and the boundary layer with Kolmogorov scaling showing the effect of limited spatial resolution. Dashed lines show the median value of $k_{1} \eta$ for which $k_{1} \ell=1$ where $\ell$ is length of hot-wire sensor. (b) The extent of the -5/3-law for the different cases.

plateaus occur, not where $R_{13}$ is zero, but in the region where it is falling toward zero. This is consistent with the findings of Saddoughi \& Veeravalli (1994) who examined a very high-Reynolds-number boundary layer flow. It should be pointed out that it is strictly possible that the fine scales may be locally isotropic even in a region where $R_{13}$ is non-zero since large scales can contribute to the shear-stress spectrum at the 

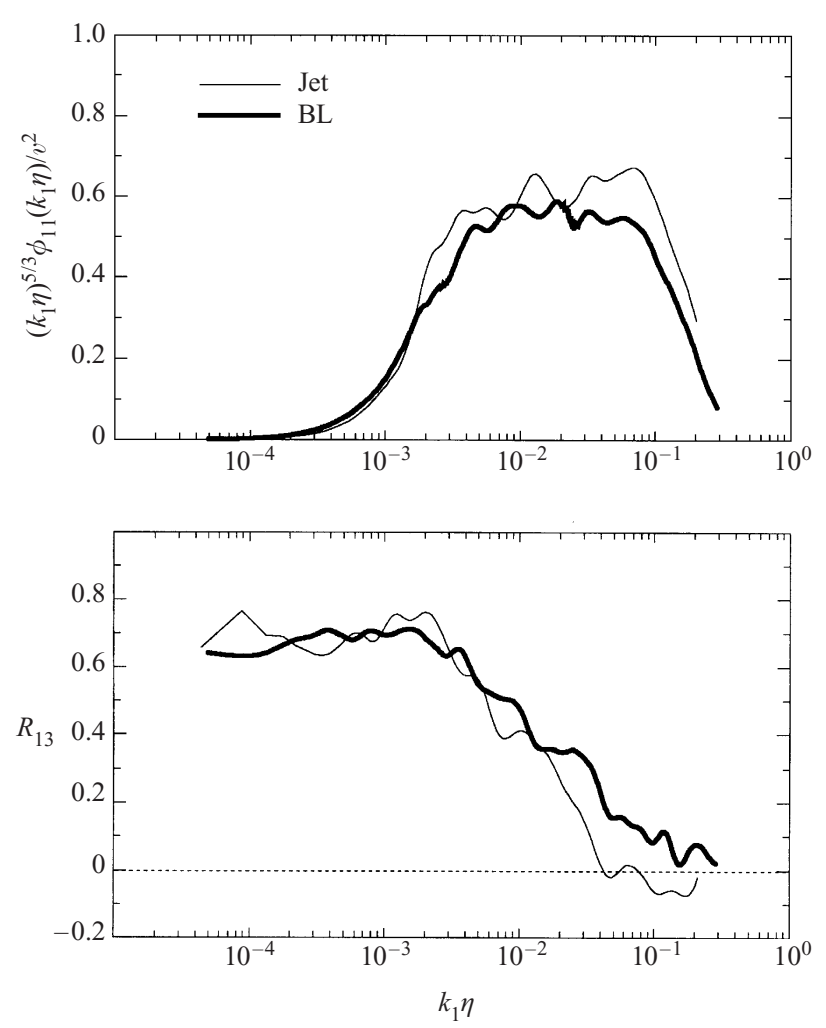

FIGURE 11. $R_{13}$ profiles together with corresponding streamwise spectra premultiplied by $\left(k_{1} \eta\right)^{5 / 3}$ for $R_{\lambda}=288$.

high-wavenumber end. This is due to the fact that if the velocity signature of an eddy is not simply sinusoidal it will contribute some energy to the spectra across a range of frequencies. Hence the small scales in the flow may in fact be locally isotropic in the region of the $-5 / 3$-law behaviour. The results from the jet model illustrate this point very well. In the model there is only one size of simple vortex structure but the Reynolds shear-stress spectrum has significant values over a range of wavenumbers.

Figure 12 shows profiles of $R_{13}$ for the two flow cases for similar Reynolds numbers and similar cross-stream positions. It can be seen that the behaviour is quite different. In particular it would seem that $R_{13}$ goes to zero much earlier in the jet case than in the boundary layer case. This suggests that the eddies which contribute to the Reynolds shear stress extend to higher wavenumber in the boundary layer, which is consistent with the trend illustrated in figure 8 . These results clearly illustrate the way in which the anisotropic large-scale eddies contribute to the Reynolds shear-stress correlation coefficient. Whilst this explanation for the non-zero $R_{13}$ has been put forward before, the models demonstrate clearly way in which the large scales contribute to the spectra at the higher wavenumbers.

\subsection{Some comments on simulation of these flows}

It is worth noting the possible implications of these results for simulation schemes for the two flows. The first point to note is that it would appear that the structure of both flows is that of large coherent vortex structures immersed in a background of fine-scale locally isotropic motions. In the case of the jet the large-scale structures 

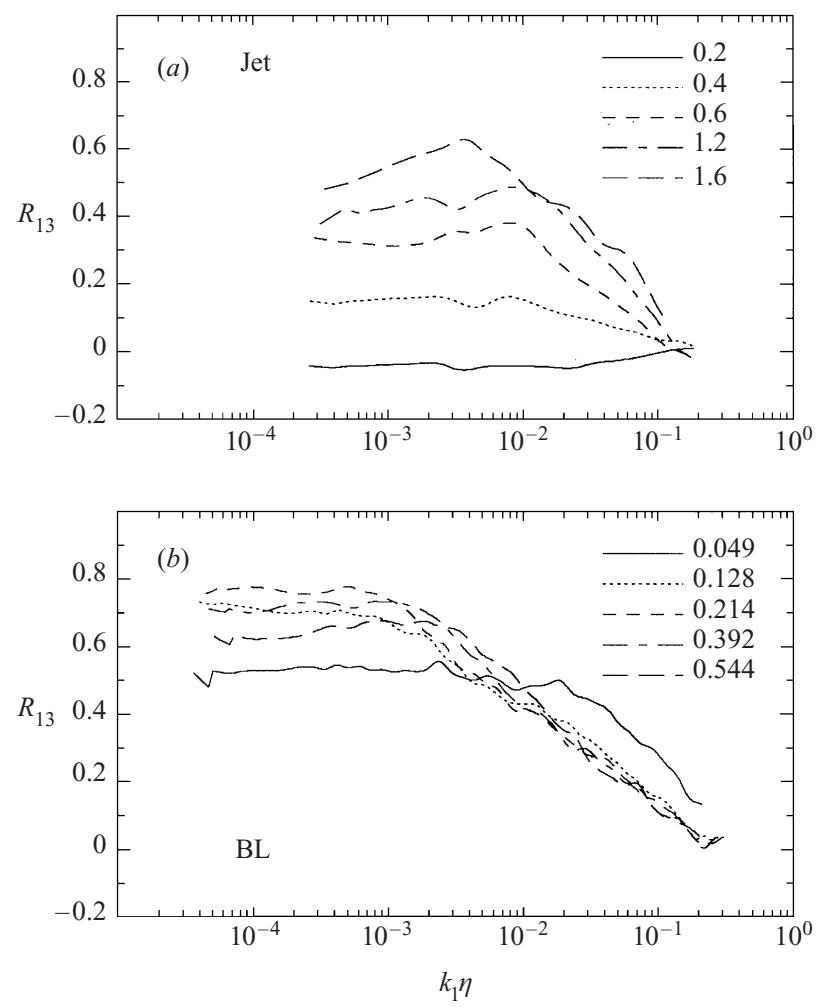

FIGURE 12. Comparison of variation of $R_{13}$ through the shear layer for $(a)$ the jet and $(b)$ the boundary layer. Non-dimensional cross-stream coordinates $\left(r / \Delta\right.$ for jet, $z / \delta_{c}$ for boundary layer) for each profile are given in the legend. $K_{\tau}=4412$ for BL; $R_{\Delta}=2384$ for jet.

seem to be of one basic size separated significantly in scale from the locally isotropic motions. This case would then seem to be ideal for schemes such as LES (large eddy simulation) since the flow has an essentially two-part structure. LES involves solving the spatially filtered Navier-Stokes and continuity equations and mathematically modelling the unresolved subgrid scales. However, the problem is not straightforward because as was shown in the numerical calculations in figure $2(b)$, the large coherent structure makes a significant contributions even at moderately high wavenumbers. Invariably in the case of LES part of this contribution will be above a preset spectral cut-off wavenumber corresponding to the filter width. In the case of the boundary layer, the problem is further complicated by the large range of scales which will be present. With this in mind, it is clear that LES sub-grid-scale models need to not only account for the universal isotropic eddy contributions but also the high-wavenumber signatures from the large-scale non-isotropic coherent structures.

The above observations suggest that it is worth pursuing alternative schemes based on approaches which can more explicitly separate locally isotropic and nonisotropic vortex structure contributions. This includes schemes which expand the velocity or vorticity fields in a set of orthonormal basis functions and then truncate the summation to retain the large-scale field. Present techniques do this also but use trigonometric terms for the basis functions. More promising basis functions include wavelets (Meneveau 1991; Dunn \& Morrison 2000) and proper orthogonal decomposition eigenfunctions (Berkooz, Holmes \& Lumley 1993; Holmes, Lumley \& 
Berkooz 1996). Ideally, basis functions derived directly from the coherent structures are required. Farge, Schneider \& Kevlahan (1999) have proposed a coherent structure based approach for two-dimensional flows which is in some respects similar to that proposed here.

\section{Conclusions}

Comparisons of spectra measured in jets and boundary layers have illustrated the difference in structure between the two flows. The Reynolds shear stress in the jet appears to be produced in a very narrow wavenumber range, whereas in the boundary layer the range is much larger. These measurements are consistent with simple coherent structure models developed by the authors. In these models the jet consists of an array of coherent structures of essentially one scale immersed in a background of fine-scale turbulence, whereas the boundary layer consists of a range of scales of similar coherent structures (also immersed in fine-scale background turbulence). It appears that the fine-scale motions are universal and locally isotropic and scale with the Kolmogorov length and velocity scales. These models present a simple physical description of the flows that provides a framework for further experimental and numerical studies.

The authors wish to dedicate this paper to the memory of Professor Tony Perry who died whilst the paper was under review. Professor Perry initiated many of the techniques and ideas that form the basis of this work and he will be greatly missed by the authors.

During this initial phase of this work the financial support of the Australian Research Council is gratefully acknowledged. During the later stages of this work, IM was supported by the National Science Foundation, through grants CTS-9983933 and ACI-9982274.

\section{REFERENCES}

Adrian, R. J., Meinhart, C. D. \& Tomkins, C. D. 2000 Vortex organization in the outer region of the turbulent boundary layer. J. Fluid Mech. 422, 1-53.

Berkooz, G., Holmes, P. \& Lumley, J. L. 1993 The proper orthogonal decomposition in the analysis of turbulent flows. Annu. Rev. Fluid Mech. 25, 539-579.

Coles, D. E. 1956 The law of the wake in the turbulent boundary layer. J. Fluid Mech. 1, 191-226.

Dunn, D. C. \& Morrison, J. F. 2000 Energy transfers in wall turbulence. Imperial College Aero. Rep. 00-01.

Erm, L. P. \& Joubert, P. N. 1991 Low Reynolds number turbulent boundary layers. J. Fluid Mech. 230, $1-44$.

Farge, M., Schneider, K. \& Kevlahan, N. 1999 Non-Gaussianity and coherent vortex simulation for two-dimensional turbulence using an adaptive othogonal wavelet basis. Phys. Fluids 11, $2187-2201$.

Holmes, P., Lumley, J. L. \& Berkooz, G. 1996 Turbulence, Coherent Structures, Dynamical Systems and Symmetry. Cambridge University Press.

Kim, K. C. \& Adrian, R. J. 1999 Very large-scale motion in the outer layer. Phys. Fluids 11, 417-422.

Kline, S. J., Reynolds, W. C., Schaub, F. A. \& Rundstadler, P. W. 1967 The structure of turbulent boundary layers. J. Fluid Mech. 30, 741-773.

MARUSIC, I. 1991 The structure of zero- and adverse-pressure gradient turbulent boundary layers. $\mathrm{PhD}$ thesis, University of Melbourne, Australia.

Marusic, I. 2001 On the role of large-scale structures in wall turbulence. Phys. Fluids 13, 735-743.

Marusic, I., Nickels, T. B. \& Perry, A. E. 1994 A comparative study of the spectra of turbulent 
jets and boundary layers. In Proc. Intl Conf. Exp. Fluid Mech. Torino, Italy. (ed. M. Onorato). Torino: Levrotto and Bella.

Marusic, I. \& Perry, A. E. 1995 A wall-wake model for the turbulence structure of boundary layers. Part 2. Further experimental support. J. Fluid Mech. 298, 389-407.

Meneveau, C. 1991 Analysis of turbulence in orthonormal wavelet representation. J. Fluid Mech. 232, 469-520.

NickeLs, T. B. 1993 Turbulent coflowing jets and vortex ring collisions. PhD thesis, University of Melbourne, Australia.

Nickels, T. B. \& Perry, A. E. 1996 An experimental and theoretical study of the turbulent coflowing jet. J. Fluid Mech. 309, 157-182.

Papoulis, A. 1965 Probability, Random Variables and Stochastic Processes. McGraw-Hill.

Perry, A. E. \& Chong, M. S. 1982 On the mechanism of wall turbulence. J. Fluid Mech. 119, $173-217$.

Perry, A. E., Henbest, S. M. \& Chong, M. S. 1986 A theoretical and experimental study of wall turbulence. J. Fluid Mech. 165, 163-199.

Perry, A. E. \& LI, J. D. 1990 Experimental support for the attached eddy hypothesis in zeropressure-gradient turbulent boundary layers. J. Fluid Mech. 218, 405-438.

Perry, A. E. \& Marusic, I. 1995 A wall-wake model for the turbulence structure of boundary layers. Part 1. Extension of the attached eddy hypothesis. J. Fluid Mech. 298, 361-388.

Saddoughi, S. G. \& Veeravalli, S. V. 1994 Local isotropy in turbulent boundary layers at high Reynolds number. J. Fluid Mech. 268, 333-372.

SreenivasAn, K. R. 1995 On the universality of the Kolmogorov constant. Phys. Fluids 7, 2778-2784. Townsend, A. A. 1976 The Structure of Turbulent Shear Flow, Vol. 2. Cambridge University Press. Zhou, J., Adrian, R. J., Balachandar, S. \& Kendall, T. M. 1999 Mechanisms for generating coherent packets of hairpin vortices in channel flow. J. Fluid Mech. 387, 353-396. 


\section{University Library}

\section{- M M N E R VA A gateway to Melbourne's research publications}

Minerva Access is the Institutional Repository of The University of Melbourne

Author/s:

Nickels, T. B.;MARUSIC, IVAN

Title:

On the different contributions of coherentstructures to the spectra of a turbulent round jetand a turbulent boundary layer

Date:

2001

Citation:

Nickels, T. B., \& Marusic, I. (2001). On the different contributions of coherentstructures to the spectra of a turbulent round jetand a turbulent boundary layer. Journal of Fluid Mechanics, 448, 367-385.

Publication Status:

Published

Persistent Link:

http://hdl.handle.net/11343/34724 